\title{
Seerosen im Palmengarten
}

Hilke Steinecke \& Dirk UllRich

\begin{abstract}
Victoria, Euryale and several other waterlilies are cultivated in the Palmengarten in the pond around the Tropicarium. Their cultivation is described.
\end{abstract}

\section{Zusammenfassung}

Im Palmengarten werden im Teich um das Tropicarium verschiedene Seerosen gehalten, darunter auch die Victoria mit ihren großen, stabilen Blättern. Kulturbedingungen für Seerosen werden genannt.

\section{Einführung}

Wenn man im Sommerhalbjahr am Wasserbecken vor dem Tropicarium vorbeikommt, fallen die vielen, üppigen Seerosen auf, die fast bis zu den ersten Frösten noch blühen.

Die Familie der Seerosengewächse (Nymphaeaceae) ist mit etwa 6 Gattungen und ca. 75 Arten relativ klein. Nymphaeaceae sind in tropischen und gemäßigten Gebieten der Welt verbreitet. Die größte und wohl auch bekannteste Gattung ist Nymphaea, zu der etwa 50 Arten gezählt werden. Für Europa werden in der Flora Europaea die vier Arten Nymphaea alba L., N. candida K. B. PresL, N. tetragona GEORGI und $N$. lotus L. angegeben. Die beiden erstgenannten kommen auch in Deutschland vor, wo sie gesetzlich geschützt sind. Nuphar lutea (L.) SM. und Nuphar pumila (TIMM) DC. sind zwei heimische, gelbblühende Teichrosen-Arten, die an NymaphaeaArten erinnern.

Die roten, gelben, weißen oder bei tropischen Nypmphaea-Arten sogar blauen Blüten geben den Seerosen einen hohen Zierwert.

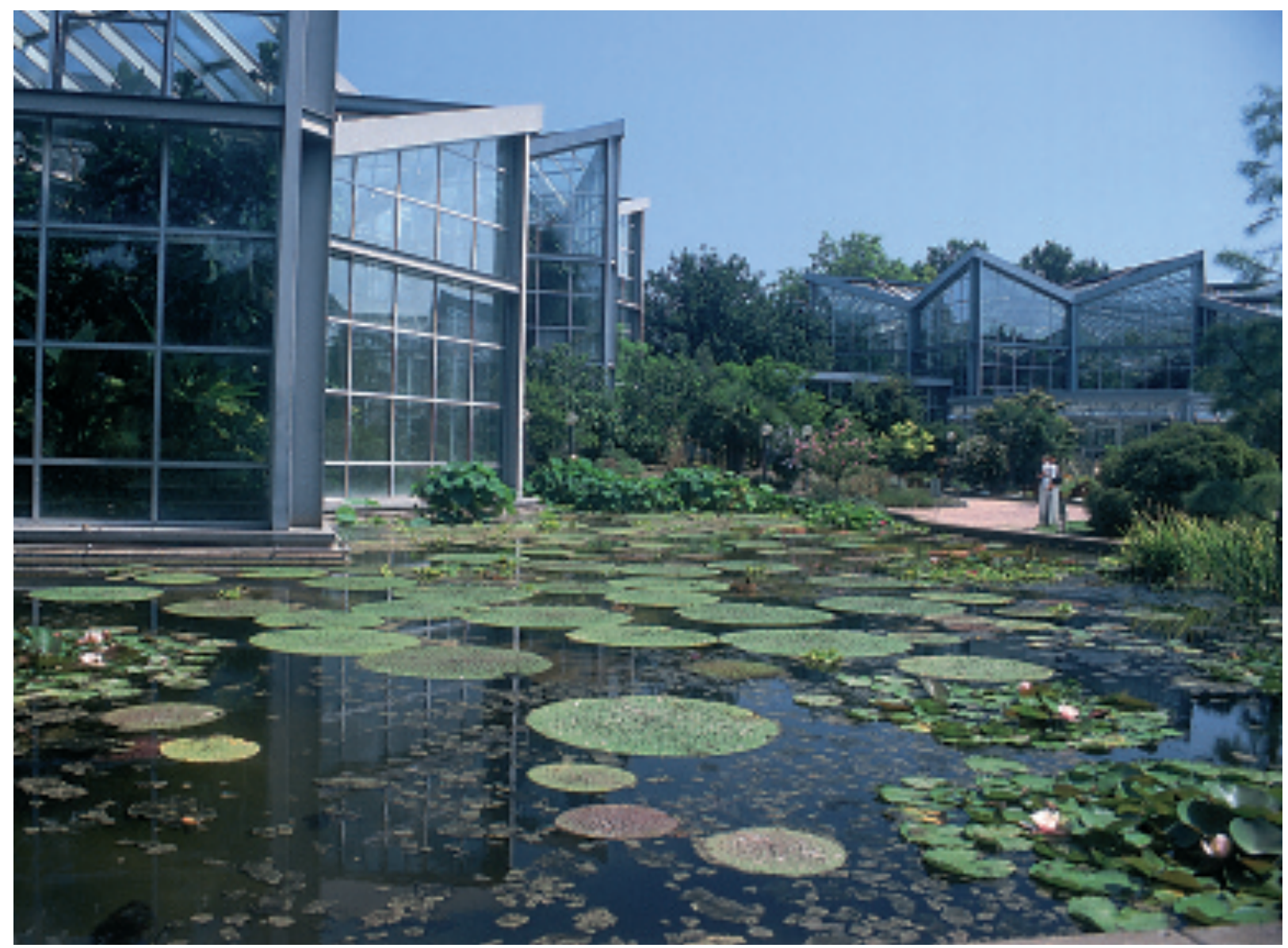


So ist es nicht verwunderlich, dass in vielen Teichen, abhängig von der Wassertemperatur, winterharte oder tropische Seerosen kultiviert werden. Das beheizte Wasserbecken vor dem Tropicarium im Palmengarten bietet für verschiedene Arten und Hybriden einen geeigneten Standort (Abb. 1)

\section{Victoria}

In dem beheizten Gewässer am Tropicarium sind die Seerosen mit ihren großen, stabilen Blättern besonders auffällig. Aus dem tropischen Südamerika stammen die beiden Arten Victoria amazonica und Victoria cruziana (Abb. 2-5). In den Jahren zwischen 1827 und 1845 wurden diese riesigen Seerosen von verschiedenen Wissenschaftlern beschrieben und nach der damaligen englischen Königin Victoria benannt. Victoria cruziana, die in Nordargentinien und Paraguay beheimatet ist, erhielt ihren Namen zu Ehren von General SANTA CRUZ, der den Botaniker ORBIGNY auf seinen Forschungsreisen begleitet hat.

Victoria regia (= V. amazonica) stammt aus Brasilien, Bolivien und British Guyana. Sie bildet riesige Blätter, die am natürlichen Standort einen Durchmesser von $2 \mathrm{~m}$ erreichen können. In Kultur bleiben sie mit einem Durchmesser von etwa $1,5 \mathrm{~m}$ kleiner. Im Freien werden die Blätter durch intensive

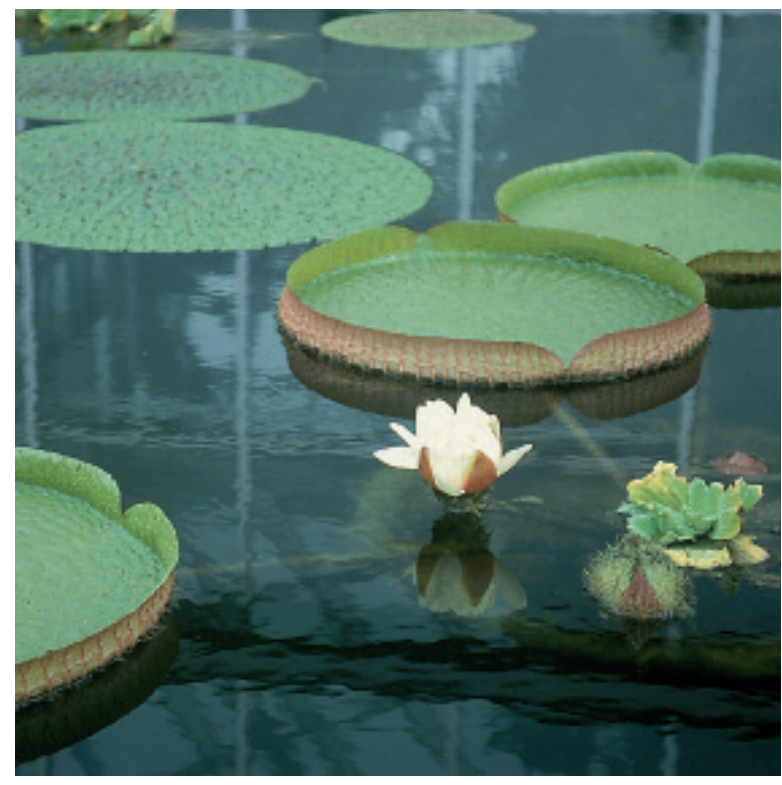

Sonneneinstrahlung ansehnlicher und stabiler als im Gewächshausbecken. Der Blattrand ist besonders markant und ähnlich wie bei einer Kuchenform etwa $5 \mathrm{~cm}$ hoch umgeschlagen. Die Rippen und der feste Rand verleihen dem Blatt eine so hohe Stabilität, dass es das Gewicht eines Kleinkindes tragen kann, ohne Schaden zu nehmen (Abb. 5). Die Konstruktion des Victoria-Blattes diente wegen seiner bewundernswerten Konstruktion als Vorbild für den Kristallpalast in London.

In ihrer Heimat ist Victoria mehrjährig. Bei uns kann $V$. regia allerdings nur als einjährige Pflanze gehalten werden und muss jedes Jahr im Januar neu ausgesät werden. Die Wassertemperatur sollte bei der Aussaat mindestens $29^{\circ} \mathrm{C}$ betragen. Dies ist am einfachsten zu gewährleisten, wenn die Seerosen im Aquarium ausgesät werden. Nach etwa drei Wochen beginnen die Samen zu keimen. Wenn sich erste Blätter und Wurzeln gebildet haben, werden

Abb. 1 (S. 119): Seerosenbecken am Tropicarium; im Hochsommer haben sich die Blätter von Victoria und Euryale sehr gut entwickelt.

Abb. 2 (links): Am ersten Tag ist die Blüte von Victoria cruziana weiß gefärbt.

Abb. 3 (rechts): Am zweiten Tag ist die Blüte rosa oder rot gefärbt.

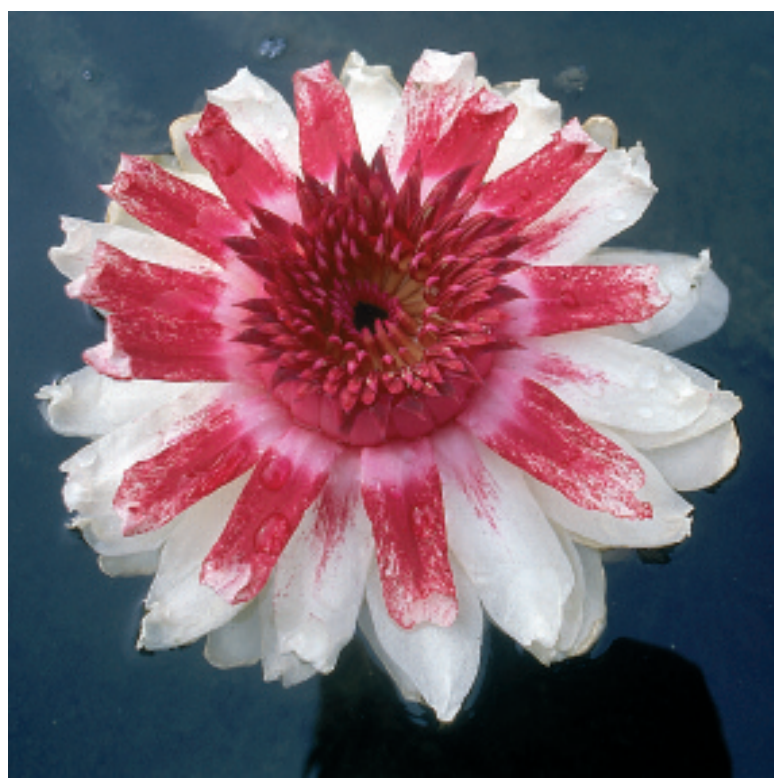




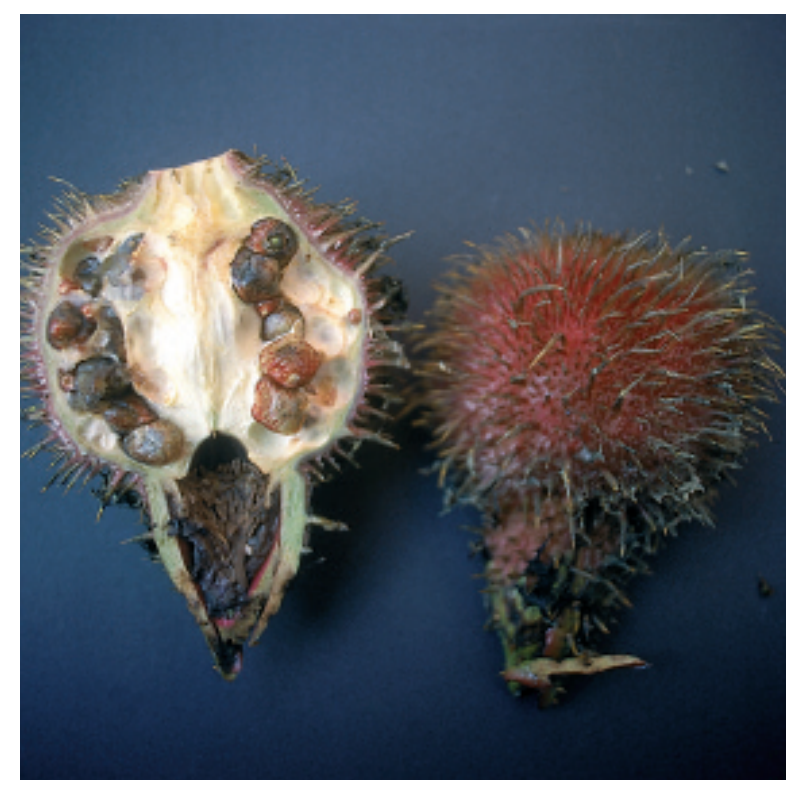

die jungen Pflanzen getopft. Eine Zusatzbeleuchtung ist während dieser Zeit unentbehrlich, da die Jungpflanzen mindestens 12 Stunden Licht pro Tag benötigen, um sich gut zu entwickeln. Bis Mitte Mai, wenn keine Nachtfröste mehr drohen, sind die Victoria-Pflanzen so weit herangewachsen, dass sie in das Außenbecken gesetzt werden können. Das mittlere Becken wird auch während des Sommers mit einer Bodenheizung gleichmäßig beheizt, die Wassertemperatur beträgt dann etwa $25{ }^{\circ} \mathrm{C}$. Victoria-Pflanzen wachsen schnell und verlangen höhere Düngergaben als andere Pflanzen. Vom Hochsommer bis in den Herbst bildet Victoria cruziana ihre ansehnlichen Blüten. Die riesigen, im Durchmesser 30-40 cm breiten, angenehm duftenden Blüten öffnen sich in der Nacht. Zunächst sind sie weiß gefärbt. Sie kontrastieren gegen den dunklen nächtlichen Hintergrund. In der natürlichen Heimat werden Käfer angelockt, die als Bestäuber fungieren. Zur Steigerung der Blütenattraktivität heizen sich die Blüten durch erhöhte Stoffwechselrate um einige Grad Celsius gegenüber der Außentempera-

Abb. 4 (links): Halbierte Frucht von Victoria cruziana.

Abb. 5 (rechts): Die Tragkraft der Blätter wird demonstriert.

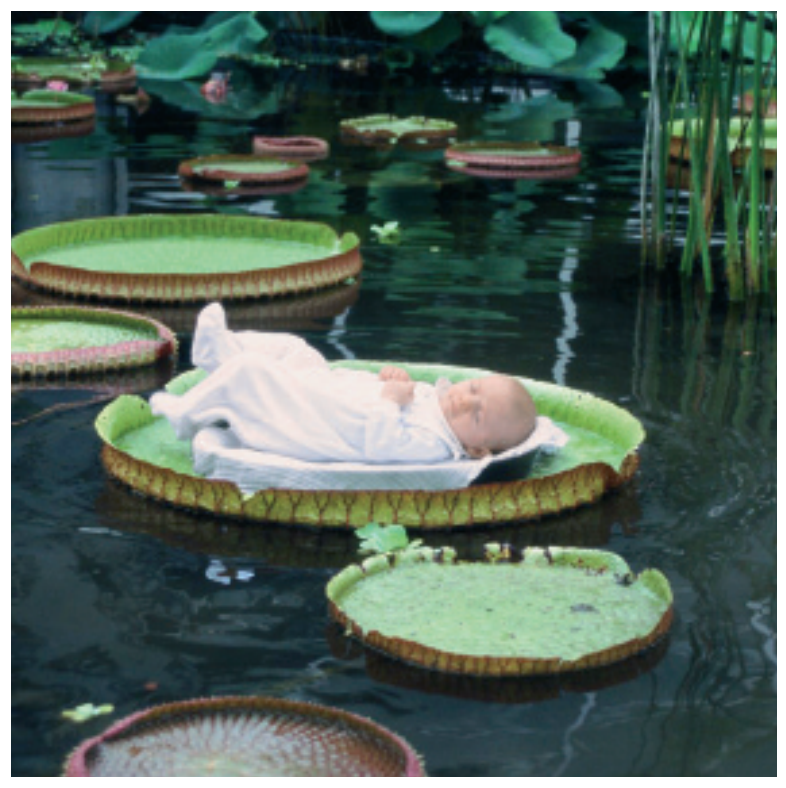

tur auf. Die Käfer fressen in den Blüten stärkehaltige Futterkörper. Während ihres Aufenthaltes in der Blüte werden die Käfer mit Pollen bepudert. Am zweiten Abend hat sich die Blüte von weiß nach rosa umgefärbt, öffnet sich erneut und die Käfer verlassen die Blüte.

Leicht mit Victoria amazonica zu verwechseln ist $V$. cruziana. Ihre Blätter sind etwas kleiner und der Rand ist etwas höher als bei V. amazonica. Die Kulturbedingungen beider Arten sind ähnlich; in Mitteleuropa wird häufig $V$. cruziana bevorzugt, da ihre Samen schon bei Wassertemperaturen um $20{ }^{\circ} \mathrm{C}$ keimen.

\section{Euryale ferox}

Im Spätsommer haben die Blätter von Euryale ferox einen großen Teil der Wasserfläche bedeckt. Sie ist die einzige Art aus der Gattung Euryale und stammt aus dem tropischen und subtropischen Ostasien, China und aus Japan. Wie bei der verwandten Victoria sind ihre Blätter mit einer Breite von 0,5-2 m ebenfalls recht groß und kräftig, jedoch ist der Blattrand nicht umgebogen. Zum Schutz vor Fraß und zur Erhöhung der Stabilität tragen die Ober- und Unterseiten der Blätter, die Blattstiele und die Blütenknospen spitze Stacheln, weshalb man von dieser Art wohl 
niemals Bilder mit einem auf dem Blatt sitzenden Kind finden wird. Die Blattunterseite ist purpurrot gefärbt. Die rötlichvioletten Blüten sind etwa $5 \mathrm{~cm}$ breit und duftend. Im Herbst bilden sich runde, ebenfalls stark bestachelte Früchte mit vielen, erbsengroßen Samen. Da diese essbar sind, wird Euryale in China angebaut; in Indien röstet man die Rhizome. Euryale lässt sich bei uns relativ leicht kultivieren, weil sie nicht so wärmebedürftig wie Victoria ist und bei Temperaturen zwischen 20 und $24^{\circ} \mathrm{C}$ wächst. Zur Anzucht ist allerdings eine Temperatur von knapp $30^{\circ} \mathrm{C}$ erforderlich.

\section{Verschiedene Nymphaea-Hybriden und -Arten}

Verschiedene Nymphaea-Hybriden mit weißen, roten oder blauen Blüten bereichern jedes Jahr das Bild der Wasserflächen rund um das Tropicarium (Abb. 7, 8). Die hier gezeigten Seerosen-Hybriden bleiben das ganze Jahr über im Becken. Wenn die Sprosse tiefer als $1 \mathrm{~m}$ unter der Wasseroberfläche liegen, sind sie nicht frostgefährdet. Gesunde, ausgewachsene Pflanzen überstehen sogar vorübergehendes Einfrieren. Sie lassen sich problemlos durch Trennen der Sprosse vermehren. Besonders hübsch ist Nymphaea $x$ daubenyana, eine kleine, fast über das ganze Jahr hinweg blau blühende, tropische Seerose. Sie wächst auch gut in kleinen Teichen oder Trögen. Dort erreicht die Pflanze allerdings nicht ihre volle Größe. Da es diese Seerose warm mag, kann man sie ebenso in geheizten Wintergärten halten oder im Sommer im Freiland, wenn der Standort sonnig und warm ist und sich das Wasser schnell aufheizt. Schon junge Pflanzen bilden an der Blattbasis Tochterpflanzen, durch die man sie leicht vermehren kann. Eine Vermehrung über Samen ist bei dieser Seerose nicht möglich, da sie unfruchtbar ist.

\section{Seerosen in Pflanzkübeln}

Seerosen sind auch eine Bereicherung für Balkon oder Terrasse. Die einfachste Art, Seerosen auf engstem Raum zu halten, sind glasierte Tongefäße, die man im Handel für ca. 50 Euro bekommen kann. Sie sollten eine Tiefe von mindestens $40 \mathrm{~cm}$ haben, besser sind 50 bis $60 \mathrm{~cm}$. Vor dem Eingang zum Mangrovenhaus des Tropicariums wurde in den letzten Jahren gezeigt, wie attraktiv Kübel mit Seerosen sein können.

Leider haben große, glasierte Gefäße meist ein Abzugsloch, das aber mit einem Silikonkleber mühelos abgedichtet werden kann. Hat man sich für einen sonnigen Platz auf dem Balkon oder der Terrasse entschieden, sollte man das Wasser einfüllen und 2 Tage stehen lassen, um sicher zu sein, dass das zugeklebte Gefäß auch wirklich dicht ist!

Anschließend kann bepflanzt werden. Am besten geeignet sind kleinwüchsige Seerosen wie z. B. Nymphaea tetragona für eine Wassertiefe bis 40 oder $50 \mathrm{~cm}$. Bevor die Seerose in den Kübel gesetzt wird, sollte sie allerdings umgetopft werden, da der im Handel übliche, mit der Pflanze mitgelieferte Topf meistens zu klein ist. Es kann ein Plastiktopf verwendet werden, der vorher mit etwas Vlies am Bodengrund abgedichtet wird, da das Substrat sonst durch die kleinen Löcher durchrieseln würde. Verwendet wird ein Gemisch aus zwei Teilen Lehm und einem Teil Landerde. Vorteilhaft ist es auch, etwas groben Sand darunter zu mischen. Wenn noch genügend Platz im Kübel vorhanden ist, sollten die Seerosen mit einigen höher wachsenden Sumpf- oder Wasserpflanzen kombiniert werden. Gut geeignet für diesen Zweck ist Papyrus (Cyperus papyrus). Falls der nun neu bepflanzte Topf zu tief im Pflanzkübel versinkt, kann er auf einen umge-

Abb. 6(S. 123, oben): Euryale ferox, in der Mitte mit einer Blüte.

Abb. 7 (S. 123, unten): Seerosen-Hybride mit Bienenbesuch.

Abb. 8 (S. 124): Seeerosen-Hybriden in verschiedensten 

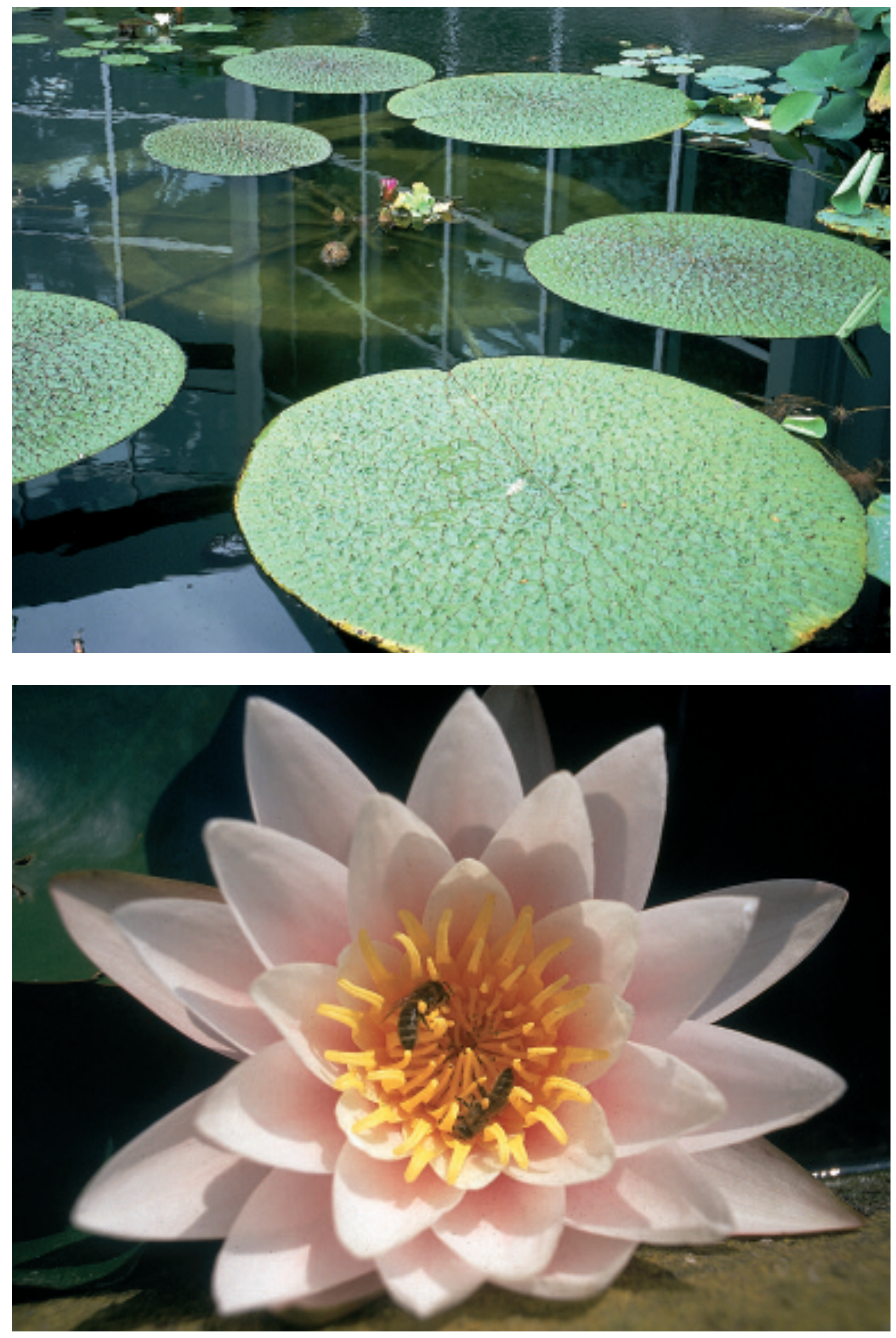
drehten und am Boden versenkten Plastiktopf gestellt werden. Auf die Wasseroberfläche kann man Schwimmpflanzen wie Wassersalat (Pistia stratiotes, Abb. 9) oder Schwimmfarn (Salvinia natans, Abb. 10) geben. Die Schwimmpflanzen sehen nicht nur schön aus, sie dienen auch der Unterdrückung des Algenwuchses.

Wenn der Kübel an einem sonnigen Ort aufgestellt wird, kann er während des Sommers viel Freude bereiten. Gedüngt werden sollte nur sparsam. Meist reicht es aus, eine Kunstdünger-Tablette nach 4 Wochen in das Erdreich zu drücken. Im Winter sollte das Gefäß geleert werden, die Seerosen samt Topf müssen aus dem Wasser genommen werden. Man bedeckt sie mit etwas Moos und stellt sie an einen kühlen Ort in den Keller. Die Seerosen verlieren alle Blätter, nur die Rhizome überwintern. Gelegentlich sollte der Topf etwas angefeuchtet werden, da sonst die Pflanze leiden oder sogar ganz vertrocknen würde. Ab Mitte März kann der Kübel wieder aufgestellt und bepflanzt werden; der kleine Miniteich füllt sich erneut mit Leben.

\section{Seerosen, typische Schwimmblattpflanzen}

Seerosen sind nicht nur hübsch anzuschauen. Mit kleinen und einfachen Experimenten können an ihnen, so auch auf Führungen im Palmengarten, typische Eigenschaften von Wasserpflanzen demonstriert werden, was gerade Kindern sehr viel Freude bereiten kann. Auf Anfrage kann man sicherlich ein paar Seerosenblätter für das nachfolgend beschriebene Experiment bekommen.

Es wird ein Blatt ausgewählt, dessen Stiel

Abb. 9 (S. 125, links): Wassersalat (Pistia stratiotes) ist ein guter Begleiter der Seerosengewächse; wie beim verwandten Aronstab ist hier im Blütenstand eine kleine Spatha ausgeprägt.

Abb. 10 (S. 125, rechts): In ein Wasserbecken gehört auch der Schwimmfarn (Salvinia). Haare auf der Blattoberseite, die wie winzige Schneebesen aussehen, lassen das Wasser von der Oberfläche abperlen.

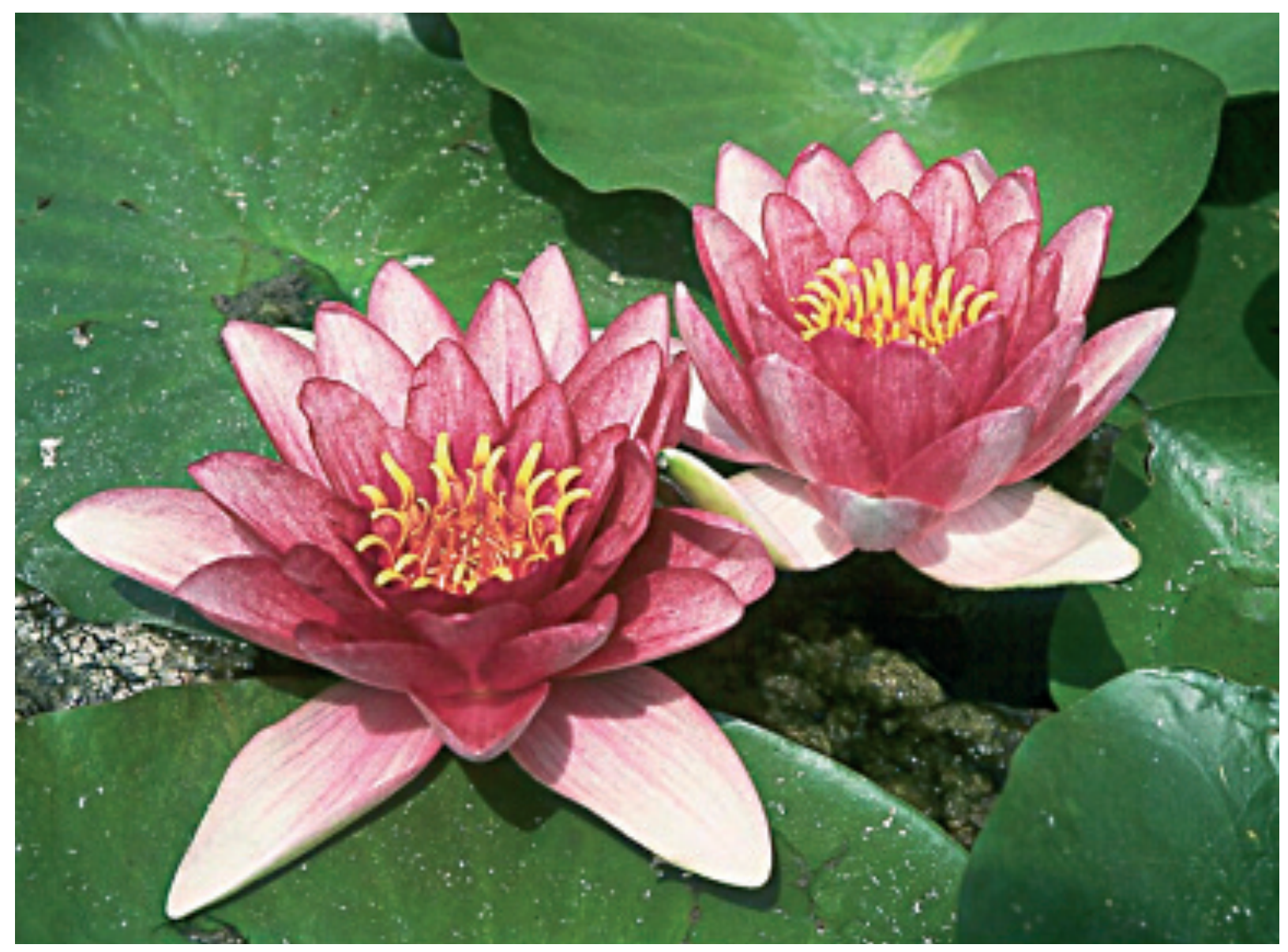




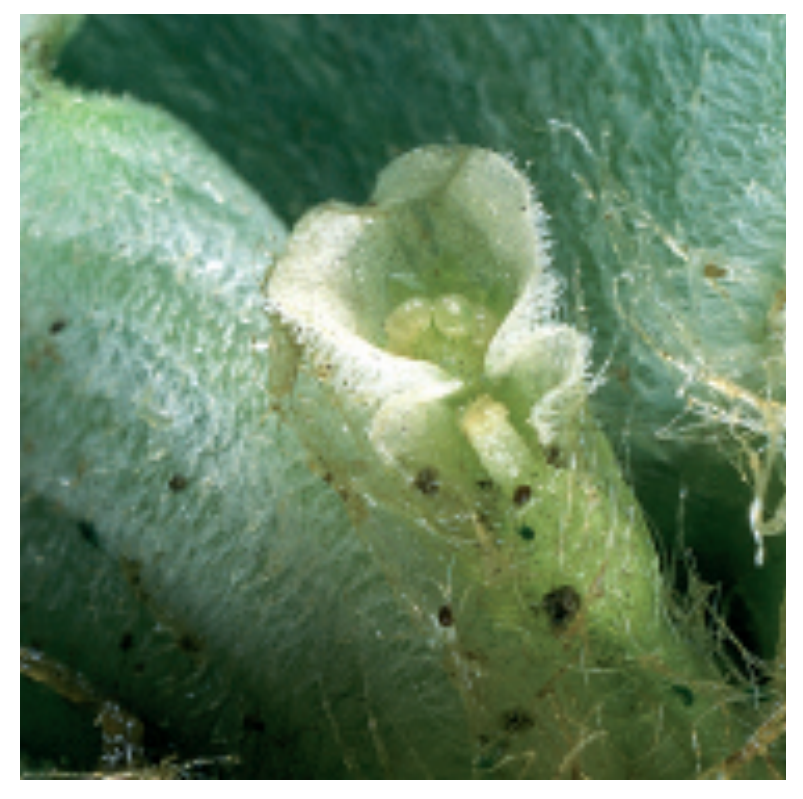

so dick ist, dass er gerade in die Ventilöffnung einer Fahrradpumpe passt. Wenn der Blattstiel in das Ventil geschoben ist (notfalls etwas festhalten), kann die Blattspreite entweder in eine große mit Wasser gefüllte Schüssel oder direkt in den Teich gehalten werden. Wenn das Blatt aufgepumpt wird, entwickeln sich auf der Oberseite viele kleine Luftbläschen. Seerosen wachsen mit ihrem Rhizom im schlecht durchlüfteten Schlamm am Grund von Teichen und Seen. Pflanzen solcher Standorte müssen deshalb den Gasaustausch im Körper verbessern, indem sie lockeres Durchlüftungsgewebe oder Hohlraumsysteme entwickeln, durch die sich im beschriebenen Experiment die Luft pumpen lässt. Luftgefüllte Organe verleihen der Pflanze zudem besseren Auftrieb und damit eine bessere Schwimmfähigkeit. Die wabenartige Konstruktion und Anordnung der Luftkanäle verursacht eine hohe Festigkeit bei nur geringem Materialeinsatz.

Der Blattstiel der Seerose ist für das Experiment besonders gut geeignet, da der Stängel mehrere, schon mit dem bloßen Auge gut erkennbare, röhrenförmige Hohlräume (Lakunen) enthält, durch die die Luft leicht

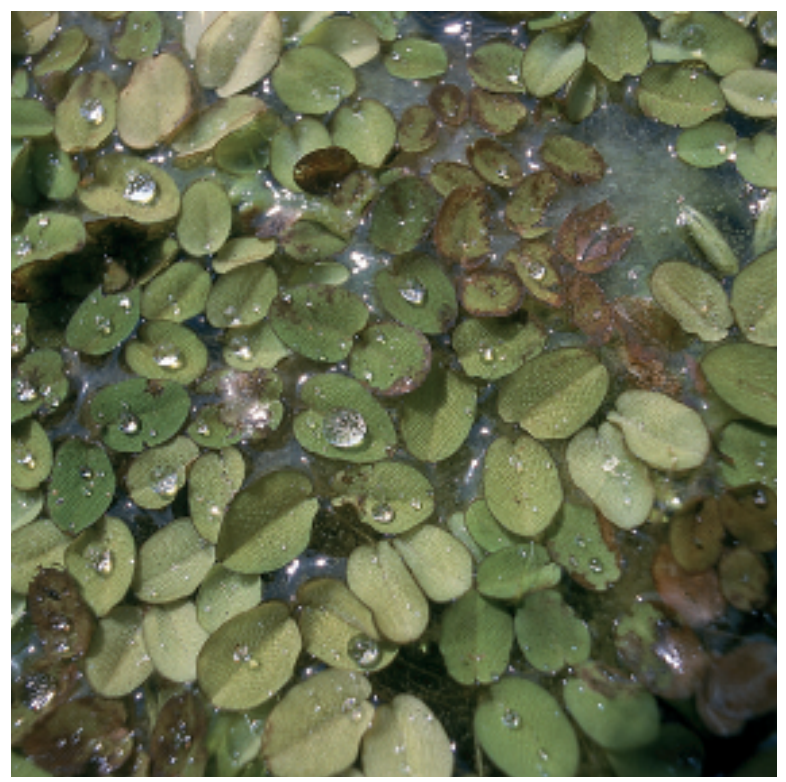

gepumpt werden kann. Die Hohlräume setzen sich bis in die Blätter fort. Auf der Blattoberfläche befinden sich zahlreiche Spaltöffnungen. Durch sie wird die Luft beim Aufpumpen nach außen gedrückt. Bei fast allen Landpflanzen dagegen befinden sich die Spaltöffnungen auf der vor Austrocknung geschützten Blattunterseite. Bei den Schwimmblättern dagegen ist es sinnvoll, dass die Spaltöffnungen nicht an der Blattunterseite im Wasser liegen, da sonst der Gasaustausch erschwert wäre.

Nach Durchführung des Experimentes kann die Blattspreite abgeschnitten und mit der Luftpumpe der Stängel „aufgepumpt“ werden. Wer es will, kann auch direkt mit dem Mund Luft durch den Stängel blasen. Im Wasser werden dabei ähnlich wie mit einem Strohhalm große, sprudelnde Luftblasen erzeugt. Falls der Seerosenstängel gut gesäubert und mit klarem Wasser durchgespült wurde, kann man ihn auch als Trinkhalm verwenden und aus einem Glas köstlichen Saft trinken.

Weitere Tipps zur Kultur von Seerosen sind WACHTER (1998) und SLOCUM \& RobiNSON (1999) zu entnehmen. 